\title{
Waveform sensors: the next challenge in biomimetic electroreception
}

\begin{abstract}
The interest in developing bioinspired electric sensors increased after the rising use of electric fields as image carriers in underwater robots and medical devices using artificial electroreception (electrotomography and electric catheterism). Electric images of objects have been most often conceived as the amplitude modulation of the local electric field on a sensory mosaic, but recent research in electric fish indicates that the evaluation of time waveform of local stimulus can increase the electrosensory channels capacities and therefore improve discrimination and recognition of target objects. This minireview deals with the present importance of developing electric sensors specifically tuned to the expected carrier waveforms to improve design of artificial bioinspired agents and diagnosis devices.
\end{abstract}

Keywords: Electroreception, Waveforms sensors
Volume 2 Issue 6 - 2017

\author{
Alejo Rodríguez Cattaneo, Angel Caputi and \\ Ana Carolina Pereira \\ Dept Neurociencias Integrativas y Computacionales, Instituto \\ de Investigaciones Biológicas Clemente Estable, Uruguay
}

Correspondence: Ana Carolina Pereira, Department of Neurociencias Integrativas y Computacionales, Instituto de Investigaciones Biológicas Clemente Estable, Av Italia 3318 Montevideo, Uruguay, CPII600, Tel 598248716I6, Email carolinapereiralarronde@gmail.com

Received: June 25, 2017 | Published: July 13, 2017

\section{Introduction}

Electroreception is a short range sensory modality that uses electric fields as a carrier of sensory signals. Artificial electric senses are bioinspired on a sensory modality expressed by aquatic vertebrates ${ }^{1-3}$ and honey bees. ${ }^{4}$ These animals use electroreceptors to measure electric fields and integrate such information in order to locate and discriminate actual (passive electroreception ${ }^{5}$ ) or virtual (corresponding to objects' polarization by a self-generated field, so called active electroreception ${ }^{6}$ ) electric sources. Research on artificial electroreception was boosted after this modality was implemented in underwater robots, ${ }^{7}$ electrotomography and intravascular catheters for medical and other purposes. ${ }^{8,9}$ This review focuses on the bioinspiring potential of research in natural electroreceptors, the present models that could be artificially implemented and the importance of waveform sensing receptive mosaics to medical and non-medical devices. Discussion is organized in three chapters dealing with electroreception in aquatic animals, natural sensors, bioinspired artificial sensors and the present models for waveforms sensing artificial sensors. Our conclusion is that developing in silico these last type of sensors is the next step in artificial electric senses.

\section{Discussion}

\section{Electroreception in aquatic animals}

Electroreception has its main expression in two main groups of weakly electric fish that have convergently evolved the ability to emit and sense electric fields, gymnotiforms from America and mormyriforms from Africa. ${ }^{10}$ In electric fish both passive and active electroreception can be considered as a part of a haptic sense that also includes lateral line mechanosensory and proprioceptive modalities. ${ }^{11}$ The rules of electric image formation have been extensively investigated. ${ }^{12-23}$ In active electroreception objects are polarized by the self-emitted field and behave as if they were emitting an objectemitted signals (as the moon appears as a light-emitter body during the night). As the self-generated electric field attenuates with a power function of distance, ${ }^{16,21,24,25}$ these object-emitted signals also decay with a power function of distance. Because of the concatenated effects of distance on the self-emitted and object-emitted fields, the perceived signal vanish when the object is placed just a few centimeters parallel to the fish's skin. ${ }^{25,26}$ In addition one must consider that objects have in general complex shapes and complex impedances. While a sphere of homogeneous conductivity illuminated by a static field can be seen as a emitting dipole, an object of complex shape can be likened as a set of dipoles including large ones depending on the volume and global impedance and small ones depending on the presence of surface irregularities. Therefore, like in touch, the set small and localized fields generated by the polarization of local edges, characteristic of rugous surfaces, can be likened as electric texture. ${ }^{23}$ Measurements of the local electric field confirmed these theoretical predictions and suggested that two cues may be used by active electroreceptive animals for object recognition: global effects (informing on object's dimension along the field lines, conductance, and position) and local effects (informing on object's surface). ${ }^{23}$

As in other haptic senses (i.e. touch or taste) three kinds of selfgenerated influences are used to pinpoint the details of explored objects. In addition to the already mentioned generation of the electric carrier, electric fish has two other ways of controlling the received signals: ${ }^{27}$ a) controlling the relative position of the sensors with respect to the explored object and b) controlling pre-receptor mechanisms in order to optimize the signal reception. These two last points result from the fact that the fish's body is itself a guide of field lines due to its conductance higher than water. ${ }^{12,22}$ Active movements of the fish may be used to orient field lines towards an explored object and to approach the skin to the object for "electric texture" analysis. ${ }^{23}$ Therefore, as mammals evaluate hardness using touch, actively electrosensing fish evaluate global aspects of objects (a variable including impedance and size). ${ }^{23}$ On the other hand "electric texture" is evaluated when object surface is located at a very short distances from the skin using dense electroreceptive mosaics present in the perioral region of the fish. ${ }^{28}$ These regions have been likened as electrosensory foveae. ${ }^{29-31}$ Importantly fish move their tails and bend their body changing the shape of the conductive path and also adapting their electroreceptive mosaic to the object that they explore. As occurs in touch when a hand grabs an object in such cases electroreception acts as part of a servo system and the shape of the object is encoded in proprioceptive signals that inform the brain about body shape. 
In the case of active electroreception, several experimental and theoretical studies in electric fish indicate that the change in waveform caused by the polarization of capacitive objects ${ }^{14,32-34}$ and differential changes in the rotational ${ }^{8,22}$ of fields differing in time waveforms and originated in different regions of the body may provide clues for discriminating object impedance, shape and location. The change in signal waveform by the presence of capacitive objects results from a transient charge of the objects, while the presence of multiple sources generating different waveforms at different distances from the object as occurs in pulse gymnotiformes might yield position dependent changes in signal waveform. In agreement with these observations, recent modeling and technical constructs have shown that capacitive sensing performs better than resistive sensing. In fact, for detecting non-conductive objects the advantage is almost an order of magnitude, while for detecting conductive objects there is a fourfold increase in performance. ${ }^{35}$ Thus, design and implementation of waveform sensing artificial electroreceptors is at the front edge in artificial electroreception research.

\section{The importance of waveform tuning in natural electroreceptors}

There are two main types of electroreceptors in nature: ampullary receptors associated with passive electroreception and tuberous electroreceptors associated with active electroreception and intraspecific communication. In general, receptor's structure consists of an epithelial capsule having a flask shape with a bulb expansion at the bottom in which either a single (gymnotiforms) ${ }^{36}$ or two (mormyriforms) $)^{37}$ sensory chambers where signal transduction is performed by sensory cells and encoding results from the spiking response of the nerve fiber that they innervate..$^{38}$ In both cases the output of natural electroreceptors are temporal series of identical nerve action potentials. The events are all or none stereotyped responses that are conducted up to the central nervous system by peripheral nerves. Thus, electric fish use one of the most robust methods (pulse interval modulation) to encode electrosensory signals. ${ }^{39}$

While in the case of ampullary output nerve fibers are spontaneously spiking in the absence of a stimulus and the spike frequency extremely sensitive to low frequency electric fields (i.e. pulse frequency modulation), tuberous electroreceptors are silent in the absence of stimuli and respond with a strong burst (i.e. pulse burst coding). ${ }^{38}$ Tuberous electroreceptors show specific responses tuned to the best frequency in the power spectral density of the self-generated stimulus, ${ }^{40,41}$ and also to different time courses even when the power spectral density of the stimulus remains constant. ${ }^{34,42}$ Tuning is important for several reasons. First, because it allows increasing the ratio between self and non-self-generated carrier electric fields and among these last fields between conspecifics and other sources. Second, changes in the amplitude or phase spectra may inform about the presence of complex impedance introduced by capacitive elements of the object. ${ }^{34,43}$ The presence of more than one type of electroreceptor with different tuning opens the possibility to perceive more than one dimension in the electric image as it occurs, for example, in color vision or tone audition (electric color, ${ }^{14,32,44}$ ). Third, in nature, receptor tuning to species-specific electric organ discharges allows the fish to detect potential mates. Males and females have seasonally modulated electric organ discharges in such a way that the sharp frequency tuning of electroreceptors parallels the changes induced by sexual hormones, ${ }^{45}$ Four, pulse gymnotiformes emit pulses resulting from the summated activity of different sources located at different places along the fish's body. ${ }^{46-48}$ Linearity suggests that whole object polarization is the sum of object polarization by such self-generated components having each a characteristic waveform. This site-dependent polarization linear effect is enhanced by the secondary polarization induced by the polarization fields coming from rest of the scene. .7,19,22 $^{-10}$ Thus, as polarization depends on source distance waveform encodes object position. ${ }^{22}$ Five, it has been recently shown that by probing capacitive objects at multiple frequencies, a quantitative framework enables decoding basic attributes of an object's composition and internal structure. ${ }^{8,35}$

\section{Artificial electric sensors}

The mathematical models of active electroreception have made significant contributions to evaluate the location and shape of an object from its physical image. The results of these mathematical models have been used to develop underwater probes and sensors able to navigate guided by this electric sense $\mathrm{e}^{49,50}$ and finding and recognizing simple objects. ${ }^{35,51,52}$ Through a dipolar approximation ${ }^{8}$ have been developed a localization algorithm and an object's shape recognition process which depend on the contribution of multi-frequency measurements ${ }^{53}$ and the movements of the source. In these models, a first-order polarization tensor describes the dependence of the equivalent dipole to the shape and complex impedance of the object. ${ }^{54}$ If this tensor is frequency-dependent and multi-frequency data are available, it is possible to extract much more information about the object than using simple amplitude data. It has been demonstrated that the multifrequency nature of the measurements in the electric field associated to the electric organ discharge are sufficient to localize an object with precision. ${ }^{8}$ However, even all the information about the object shape is contained in the polarization tensor once the object is located by a multi-frequency analysis, it is not possible to determine it from a single measure of the transcutaneous field. Probably, the fish solve this problem actively swimming around the prey and extracting measures since the successive fish's positions ${ }^{55}$ and therefore multisensor arrays should be the most efficient for artificial and natural electric senses.

Moreover, the electric measurement of tissue impedance for medical uses (electrical impedance tomography) is known since 1978 (for a review on principles and applications see ${ }^{56}$ ). It has some advantages and disadvantages over conventional X-ray tomography. It is cheaper and safer since it does not require any kind of ionizing radiation. Stimulus strength are below the threshold at which they would cause significant stimulation of excitable tissues as heart, muscles, or nerves and thermal effects are small and diffused over the body. Stimulus frequency is sufficiently high not to cause electrolytic effects in the body. The feasibility of this technique for lung imaging was first shown by Cheney et al. ${ }^{57}$ who develop an algorithm for solving the inverse conductivity problem at the thoracic level. This technique may provide information in order to supply lung protective ventilation to patients during critical illness or anesthesia. This qualify electro-impedance tomography to be continuously used at the bedside in intensive care unit for monitoring ventilatory activity. Although electro-impedance tomography has much less spatial resolution to explore anatomical lesions than conventional X-ray tomography, it has several promising advantages for exploring functional aspects. Because of its large temporal resolution it could combine ventilatory and hemodynamic recording $5^{58}$ and its further development might allow simultaneous visualization of both regional blood flow and ventilation, enabling the evaluation of local mismatches between lung ventilation and perfusion. Finally, several non-medical uses of electro 
impedance measurements has been proposed. The same principles already described can be applied to explore geological structures.

While other medical uses have been proposed, including differential diagnosis of tumours, perhaps the newest innovation is its use in vascular diagnosis. ${ }^{9}$ This idea combines the ability of active electroreception to evaluate surface texture with the possibility to detect capacitive elements as plates of atheroma due to the deposit of cholesterol. In this case, the authors tested two models: the first with one ring electrode which can obtain dynamic one-dimension electric images and the second with $16(4 \times 4)$ ring electrodes which can obtain dynamic and static electric images. They used pulsatile stimuli either biomimetic (with a waveform similar to the electric organ discharge of an electric fish) or non-mimetic (using a spectrum with a peak power frequency of $10 \mathrm{KHz}$ ). They evaluated both the change in peak to peak amplitude of the signal and the change in the time course of the stimulus waveform. Synthetic plaques were detected and localized using both types of stimuli but the non-biomimetic stimulus was the most efficient when it was used to explore a natural arteria and artificial plaque suggesting that high frequencies are better for exploring arteries. Change in waveform yielded the most informative signal.

They concluded that for single electrode images the strongest correlating parameter is the relative core area and it is possible to assess the position and the angle of a synthetic plaque within the artery wall through the peak modulations of static and dynamic amplitude and the waveform images. Finally, due to the short range and precision active artificial electroreception can have industrial uses for evaluating changes in electric texture due to the effects of rust and corrosion in the inner surfaces of drainage, water, and oil pipes and also external tubular devices as the legs of oil platforms anchored directly onto the seabed where sunlight has very poor access.

\section{Models for waveform detection and their potential implementation}

The mechanisms of waveform tuning of natural electroreceptors are not well understood. Nonetheless, the asymmetry between the impedance of the plug and the wall capsula and the dynamic properties of sensory cells appear to be the main factors in waveform detection. Resonance of sensory cells has been involved in such tuning. Electroreceptor modeling has been made first by Bennett ${ }^{59}$ on the basis on his observation of damped oscillation in response to electric stimuli. This qualitative model has been further developed to explain how Mormyriforms and Gymnotiforms specifically detect capacitance-induced changes in EOD waveform.

In mormyriformes receptors consist of two sensory chambers connected through a small opening (Figure 1A). In the outer chamber, sensory cells are pure amplitude coders. ${ }^{32,60,61}$ In the inner chamber sensory cells respond not only to signal amplitude but they are also extremely sensitive to EOD waveform distortions, ${ }^{42,60,61}$ firing with shorter latency and an increased number of spikes per burst, even if the EOD amplitude has been kept constant. The core of Shuai et al. ${ }^{62}$ model are the differences in the dynamic of the sensory cell membranes at the inner and outer receptor chambers. They postulate that sensory cells in the outer chamber have a simple resistivecapacitive membrane equivalent circuit, while in the sensory cells of the inner chamber the stimulus evokes a calcium-mediated depolarizing potential which counterbalanced after a short delay by the opening of calcium-activated potassium channels. The calcium concentration increase due to calcium channel opening links the opening probability of neighbor calcium and potassium channels causing membrane resonance and therefore changes in electroreceptor responsiveness to different waveform. ${ }^{63}$

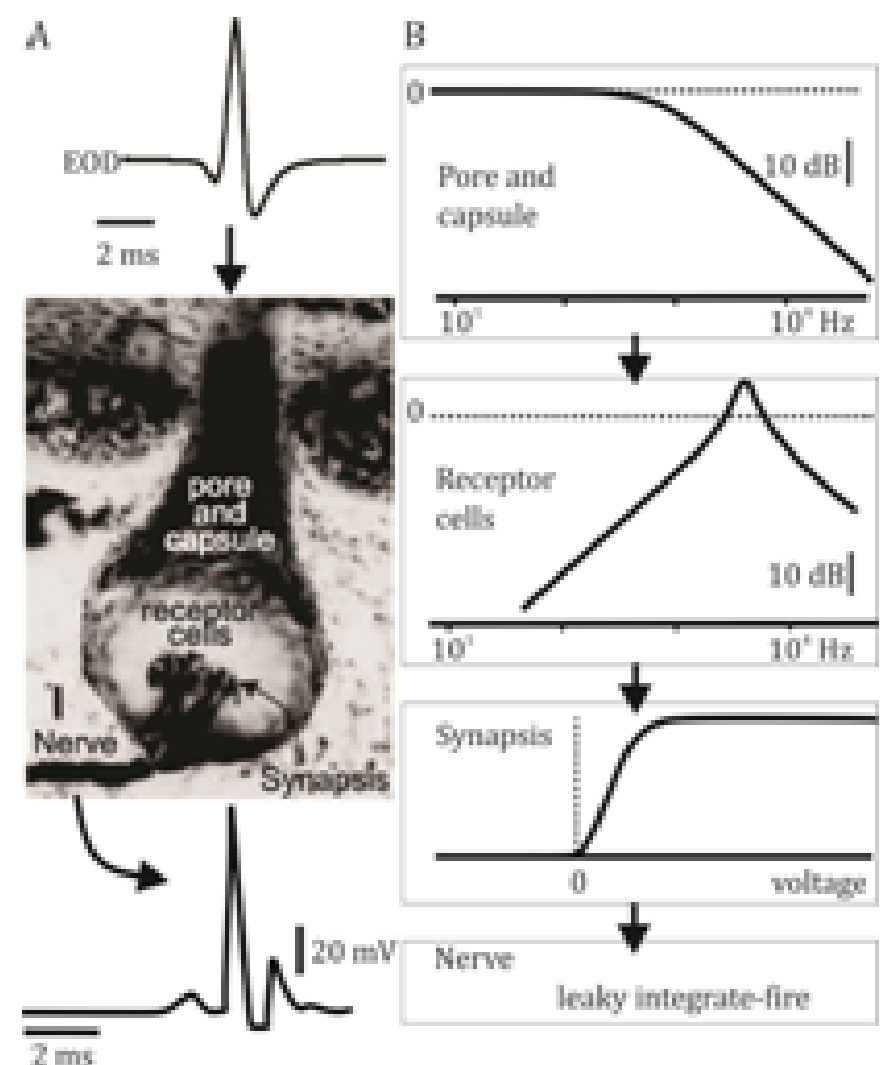

Figure I Computational model of an electroreceptor. (A) A micrograph of a tuberous electroreceptor showing input (EOD: electric organ discharge) and output signals. (B) Four modules connected in series constitute the artificial electroreceptor: a) pre-receptor structures (pore and capsule) connecting the lumen of the organ with water are modeled as a low pass filter whose cut off frequency depend on the organ's diameter; b) a group of receptor cells transduces the electrical stimulus and is modeled as a second order differential equation yielding a band pass filtered ringing response; $c$ ) a synaptic module rectifying receptor response; and d) a leaky-integrate-and-fire model corresponds to a neural encoding module, modelating the peripheral tree of terminals of a single nerve fiber synaptically in contact with the group of transducer cells.

Cilleruelo and Caputi's extended Bennett's model for gymnotiformes (Figure 1B ${ }^{64}$ ) suggested an electronic implementation using discrete elements. ${ }^{65}$ This test of function suggested that the same algorithms and structure can be implemented using integrated nanotechnologies. This artificial electroreceptor prototype consisted of four modules connected in series. The first module accounted for pre-receptor structures including the capsule and the pore connecting the lumen of the organ with water. This was modeled as a low pass filter having a cut off frequency depending on the parameters that determine $\tau$ (the diameter of the organ and the podre and the material and thickness of the capsula).

\section{$d \operatorname{Vin} / d t+\operatorname{Vin} / \tau=$ Vout $/ \tau$}

The second module accounted for transductor cells in which each is considered as a second order linear system. Taking into account that 
many receptor cells converge on the afferent nerve the input on the nerve results from integrating the contribution of individual receptor cells.

$d 2 V r / d t 2+2 \cdot \zeta \cdot \omega 0 \cdot(1+\kappa i) d V r / d t+(\omega 0 \cdot(1+\kappa i)) 2 \cdot V r=d V i n / d t$

While the low pass module introduces an attenuation of high frequencies, the resonant module has a transfer function described as a tent-shaped Bode plot whose peak is mainly dependent on the resonance frequency and whose slopes in the neighborhood of the peak depend on the attenuation factor. The output of receptor cells are then rectified by a synaptic-like module similar to an electronic diode and transformed in a burst of discrete events using a leaky integratefire-and-reset circuit. ${ }^{66}$ This model is able to reproduce experimental performance of electroreceptors observed experimentally $y^{34,40,41,59}$ and can be easily implemented electronically.

\section{Conclusion}

Electric images sensed by electrosensory mosaics may provide information of material, shape and location of nearby objects, particularly in the case of active senses. The above discussion have shown the importance of selective sensing the carrier waveform by tuned electroreceptor, the potential use in artificial devices and the potential implementation of such sensors in silico. In addition, texture evaluation requires receptive mosaics having a dense array of waveform sensors. As the shape of artificial agents may be selfdeformed to polarize and observe the target objects from different points of view, flexibility in the design of sensory mosaics is required. We conclude that the next challenge in artificial electroreception is to build flexible electroreceptive sensory mosaics composed of dense arrays of waveform sensitive elements at the nanoscale level.

\section{Acknowledgements}

Authors thank Lic. Lucia Osorio for English editing.

\section{Conflict of interest}

The author declares no conflict of interest.

\section{References}

1. Lissmann HW. On the function and evolution of electric organs in fish Journal of Experimental Biology. 1958;35(1):156-191.

2. Fritzsch B, Münz H. Electroreception in amphibians. In: Bullock TH, et al. editors. Electroreception. USA: Wiley; 1986. p. 483-496.

3. Pettigrew JD. Electroreception in monotremes. $J$ Exp Biol. 1999;202(Pt10):1447-1454.

4. Clarke D, Whitney H, Sutton G, et al. Detection and learning of floral electric fields by bumblebees. Science. 2013;340(6128):66-69.

5. Kalmijn AJ. The detection of electric fields from inanimate and animate sources other than electric organs. In: Fessard A editors. Handbook of sensory physiology, Vol III/3: Electroreceptors and Other Specialized Receptors in Lower Vertrebrates. Germany: Springer-Verlag; 1974. p. 147-200.

6. Bennett MVL. Electric organ. In: Hoar WS, Randall DJ editors. Fish physiology. (Vol V), England, UK: Academic Press; 1971. p. 347-491.

7. Boyer F, Lebastard V. Exploration of objects by an underwater robot with electric sense. Biomimetic and biohybrid systems. 2012. p. 50-61.

8. Ammari H, Boulier T, Garnier J, et al. Mathematical modelling of the electric sense of fish: the role of multi-frequency measurements and movement. Bioinspiration \& Biomimetic. 2017;12(2):025002.
9. Gottwald M, Matuschek A, Von Der Emde G. An active electrolocation catheter system for imaging and analysis of coronary plaques. Bioinspiration \& Biomimetics. 2017;12(1) 015002.

10. Moller P. Electric fishes: history and behavior. (Vol 17), UK: Chapman, Hall; 1995. 584 p.

11. Caputi AA, Aguilera PA, Pereira AC, et al. On the haptic nature of the active electric sense of fish. Brain Res. 2013;1536:27-43.

12. Caputi A, Budelli R. The electric image in weakly electric fish: I. A databased model of waveform generation in Gymnotus carapo. Journal of Computational Neuroscience. 1995;2(2):131-147.

13. Caputi AA, Budelli R, Grant K, et al. The electric image in weakly electric fish: physical images of resistive objects in Gnathonemus petersii. J Exp Biol. 1998;201(Pt 14):2115-2128.

14. Budelli R, Caputi AA. The electric image in weakly electric fish: perception of objects of complex impedance. Journal of Experimental Biology. 2000;203(3):481-492.

15. Rother D, Migliaro A, Canetti R, et al. Electric images of two low resistance objects in weakly electric fish. Biosystems. 2003;71(1):169177.

16. Chen L, House JL, Krahe R, et al. Modeling signal and background components of electrosensory scenes. Journal of Comparative Physiology A. 2005;191:331-345.

17. Migliaro A, Caputi AA, Budelli R. Theoretical analysis of pre-receptor image conditioning in weakly electric fish. PLoS Computational Biology. 2005;1(2):16.

18. Nelson ME. Target detection, image analysis, and modeling. In: Bullock TH, et al. editors. Electroreception, USA: Springer; 2005. p. 290-317.

19. Pereira AC, Centurión V, Caputi AA. Contextual effects of small environments on the electric images of objects and their brain evoked responses in weakly electric fish. J Exp Biol. 2005;208(Pt 5):961-972.

20. Caputi AA, Budelli R. Peripheral electrosensory imaging by weakly electric fish. J Comp Physiol A Neuroethol Sens Neural Behav Physiol. 2006;192(6):587-600.

21. Nelson ME, MacIver MA. Sensory acquisition in active sensing systems. J Comp Physiol A Neuroethol Sens Neural Behav Physiol. 2006;192(6):573-586.

22. Pereira AC, Caputi AA. Imaging in electrosensory systems. Interdisciplinary Sciences: Computational Life Sciences. 2010;2(4):291307.

23. Caputi AA, Aguilera PA, Pereira AC. Active electric imaging: body-object interplay and object's “electric texture". PloS one. 2011;6(8):e22793.

24. Knudsen EI. Spatial aspects of the electric fields generated by weakly electric fish. Journal of Comparative Physiology. 1975;99(2):103-118.

25. Pereira AC, Aguilera P, Caputi AA. The active electrosensory range of Gymnotus omarorum. Journal of Experimental Biology. 2012;215(18):3266-3280.

26. Caputi AA, Castelló ME, Aguilera PA, et al. Active electroreception in Gymnotus omari: imaging, object discrimination, and early processing of actively generated signals. Journal of Physiology-Paris. 2008;102(46):256-271.

27. Caputi AA. Contributions of electric fish to the understanding sensory processing by reafferent systems. Journal of Physiology-Paris. 2004;98(1-3):81-97.

28. Harder W, Schief A, Uhlemann H. Zur Empfindlichkeit des schwachelektrischen Fisches Gnathonemus petersii (Gthr. 1862) (Mormyriformes, Teleostei) gegenüber elektrischen Feldern. Zeitschrift für vergleichende Physiologie. 1966;54(1):89-108. 
29. Castelló ME, Aguilera PA, Trujillo Cenoz O, et al. Electroreception in Gymnotus carapo: pre-receptor processing and the distribution of electroreceptor types. Journal of Experimental Biology. 2000;203(21):3279-3287.

30. Caputi AA, Castelló ME, Aguilera P, et al. Electrolocation and electrocommunication in pulse gymnotids: signal carriers, pre-receptor mechanisms and the electrosensory mosaic. J Physiol Paris. 2002;96(56):493-505.

31. Bacelo J, Engelmann J, Hollmann M, et al. Functional foveae in an electrosensory system. J Comp Neurol. 2008;511(3):342-359.

32. Von Der Emde G. Discrimination of objects through electrolocation in the weakly electric fish, Gnathonemus petersii. Journal of Comparative Physiology A. 1990;167(3):413-421.

33. Aguilera PA, Caputi AA. Electroreception in G. carapo: detection of changes in waveform of the electrosensory signals. Journal of experimental biology. 2003;206:989-998.

34. Rodríguez-Cattaneo A, Aguilera PA, Caputi AA. Waveform sensitivity of electroreceptors in the pulse-type weakly electric fish Gymnotus omarorum. J Exp Biol. 2017;220(Pt9):1663-1673.

35. Bai Y, Neveln ID, Peshkin M, et al. Enhanced detection performance in electrosense through capacitive sensing. Bioinspir Biomim. 2016;11(5):055001.

36. Szabo T. Anatomy of the specialized lateral line organs of electroreception In: Fessar A editors. Germany: Springer-Verlag Berlin; 1974. p. 13-58.

37. Bell CC, Zakon H, Finger TE. Mormyromast electroreceptor organs and their afferent fibers in mormyrid fish: I. Morphology. J Comp Neurol. 1989;286(3):391-407.

38. Zakon HH. The electroreceptive periphery. In: Bullock THW, Heiligenberg W editors. Electroreception. USA: Wiley; 1986. p. $103-$ 156

39. Bendat JS, Piersol AG. Random data: analysis and measurement procedures. 4th ed. USA: John Wiley, Sons; 2011. 640 p.

40. Bastian J. Frequency response characteristics of electroreceptors in weakly electric fish (Gymnotoidei) with a pulse discharge. Journal of Comparative Physiology. 1976;112(2):165-180.

41. Mc Kibben JR, Hopkins CD, Yager DD. Directional sensitivity of tuberous electroreceptors: polarity preferences and frequency tuning. Journal of Comparative Physiology A. 1993;173(4):415-424.

42. Von Der Emde G, Bleckmann H. Waveform tuning of electroreceptor cells in the weakly electric fish, Gnathonemus petersii. Journal of Comparative Physiology A. 1997;181(5):511-524.

43. Aguilera PA, Caputi AA. Electroreception in G. carapo: detection of changes in waveform of the electrosensory signals. $J$ Exp Biol. 2003;206(Pt 6):989-998

44. Meyer JH. Behavioral responses of weakly electric fish to complex impedances. Journal of comparative physiology. 1982;145(4):459-470.

45. Meyer JH, Zakon HH. Androgens alter the tuning of electroreceptors. Science. 1982;217(4560):635-637.

46. Caputi AA. The electric organ discharge of pulse gymnotiforms: the transformation of a simple impulse into a complex spatio-tempora electromotor pattern. J Exp Biol. 1999;202(Pt10):1229-1241.

47. Rodríguez-Cattaneo A, Pereira AC, Aguilera PA, et al. Species-specific diversity of a fixed motor pattern: the electric organ discharge of Gymnotus. PLoS One. 2008;3(5):e2038.

48. Rodríguez-Cattaneo A, Aguilera P, Cilleruelo E, et al. Electric organ discharge diversity in the genus Gymnotus: anatomo-functional groups and electrogenic mechanisms. Journal of Experimental Biology. 2013;216(8):1501-1515
49. Lebastard V, Chevallereau C, Girin A, et al. Environment reconstruction and navigation with electric sense based on a kalman filter. The International Journal of Robotics Research. 2013;32(2):172-188.

50. Solberg JR, Lynch KM, MacIver MA. Active electrolocation for underwater target localization. The International Journal of Robotics Research. 2008;27(5):529-548.

51. Bai Y, Snyder JB, Peshkin M, et al. Finding and identifying simple objects underwater with active electrosense. The International Journal of Robotics Research. 2015;34(10):1255-1277.

52. Lanneau S, Lebastard V, Boyer F. Object shape recognition using electric sense and ellipsoid's polarization tensor. Robotics and Automation (ICRA) 2016 IEEE International Conference on; Sweden: Springer; 2016. p. 4692-4699.

53. Ammari H, Wang H. Time-domain multiscale shape identification in electro-sensing. 2016. p 23-45.

54. Ammari H, Kang H. Polarization and moment tensors: With applications to inverse problems and effective medium theory. (Vol 162), USA: Springer Science \& Business Media; 2007. 314 p.

55. Ammari H, Boulier T, Garnier J, et al. Shape recognition and classification in electro-sensing. Proceedings of the National Academy of Sciences. 2014;11(32):11652-11657.

56. Holder DS. Electrical impedance tomography: Methods, history and applications. USA: CRC Press; 2004. 456 p

57. Cheney M, Isaacson D, Newell JC, et al. NOSER: An algorithm for solving the inverse conductivity problem. International Journal of Imaging Systems and Technology. 1990;2(2):66-75.

58. Solà J, Adler A, Santos A, et al. Non-invasive monitoring of central blood pressure by electrical impedance tomography: first experimental evidence. Med Biol Eng Comput. 2011;49(4):409-415.

59. Bennett MVL. Mechanisms of electroreception. In: Cahn P, editors. Lateral Line Detectors. USA: Indiana University press; 1967. p. 313 393.

60. Bell CC. Mormyromast electroreceptor organs and their afferent fibers in mormyrid fish. II. Intra-axonal recordings show initial stages of central processing. J Neurophysiol. 1990;63(2):303-318.

61. Von Der Emde G, Bleckmann H. Differential responses of two types of electroreceptive afferents to signal distortions may permit capacitance measurement in a weakly electric fish, Gnathonemus petersii. Journal of Comparative Physiology A. 1992;171(5):683-694.

62. Shuai J, Kashimori Y, Kambara T. Electroreceptor model of the weakly electric fish Gnathonemus petersii. I. The model and the origin of differences between A-and B-receptors. Biophysical J. 1998;75(4):17121726 .

63. Shuai J, Kashimori Y, Hoshino O, et al. Electroreceptor model of weakly electric fish Gnathonemus petersii: II. Cellular origin of inverse waveform tuning. Biophysical journal. 1999;76(6):3012-3025.

64. Cilleruelo ER, Caputi AA. Encoding electric signals by Gymnotus omarorum: heuristic modeling of tuberous electroreceptor organs. Brain research. 2012;1434:102-114.

65. Caputi AA, Ferreira JA, Cilleruelo ER. Modulo detector, transductor y codificador de señales eléctricas bioinspirado en los peces eléctricos. Patent, Uruguay: Springer; 2011

66. Abbott LF. Lapique's introduction of the integrate and fire model neuron (1907). Brain Research Bulletin. 1999;50:303-304. 